\title{
Importance of Protamine Phosphorylation to Histone Displacement in Spermatids: Can the Disruption of this Process be used for Male Contraception?
}

\author{
R. Balhorn \\ N.V. Hud \\ M. Corzett \\ J. Mazrimas
}

June 1995

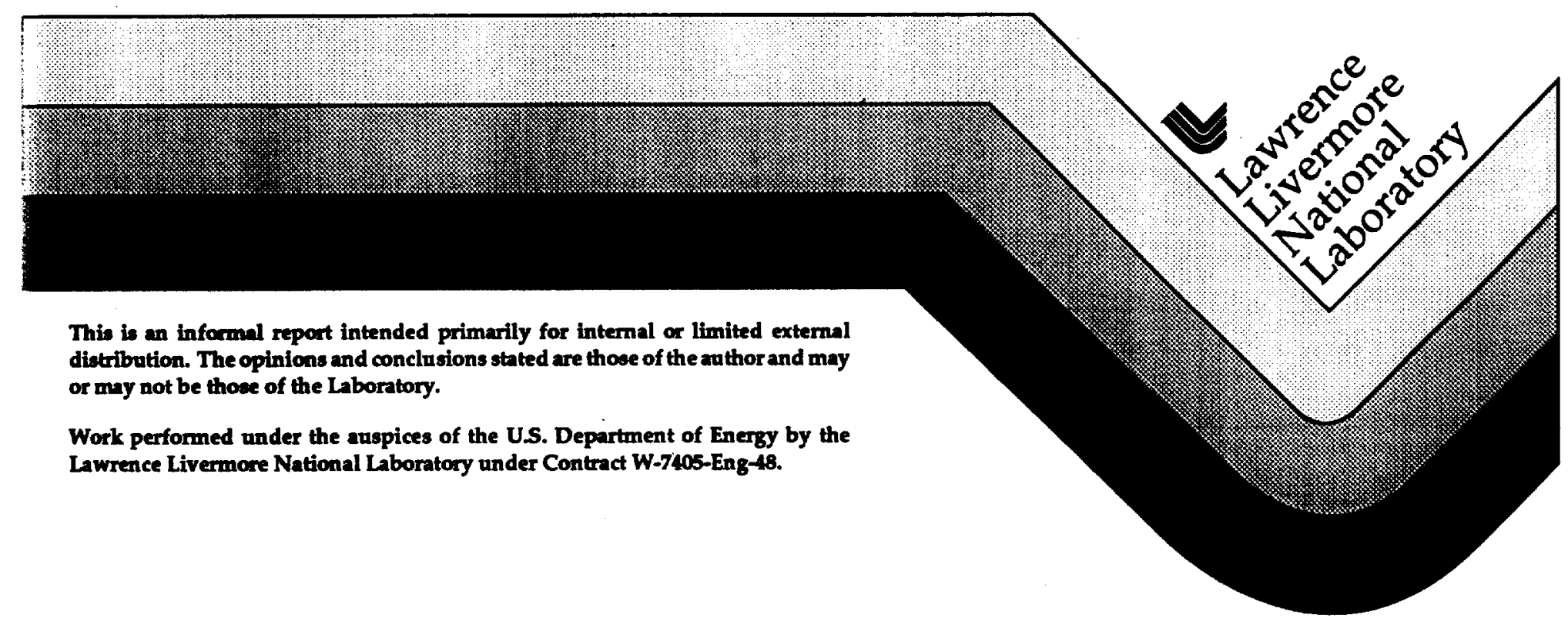




\section{DISCLAIMER}

This document was prepared as an account of work sponsored by an agency of the United States Government. Neither the United States Government nor the University of California nor any of their employees, makes any warranty, express or implied, or assumes any legal liability or responsibility for the accuracy, completeness, or usefulness of any information, apparatus, product, or process disclosed, or represents that its use would not infringe privately owned rights. Reference herein to any specific commercial product, process, or service by trade name, trademark, manufacturer, or otherwise, does not necessarily constitute or imply its endorsement, recommendation, or favoring by the United States Government or the University of California. The views and opinions of authors expressed herein do not necessarily state or reflect those of the United States Government or the University of California, and shall not be used for advertising or product endorsement purposes.

This report has been reproduced directly from the best available copy.

Available to DOE and DOE contractors from the Office of Scientific and Technical Information

P.O. Box 62, Oak Ridge, TN 37831

Prices available from (615) 576-8401, FTS 626-8401

Available to the public from the

National Technical Information Service

U.S. Department of Commerce

5285 Port Royal Rd.,

Springfield, VA 22161 


\section{Importance of Protamine Phosphorylation to Histone Displacement in Spermatids: Can the Disruption of this Process be used for Male Contraception?}

R. Balhorn, N.V. Hud, M. Corzett and J. Mazrimas

Biology and Biotechnology Research Program Lawrence Livermore National Laboratory 


\section{ABSTRACT}

Protamine is a small protein that packages DNA in the sperm of most vertebrates. Shortly after its synthesis, the serine and threonine residues in each protamine molecule are phosphorylated and the modified proteins are deposited onto DNA, displacing the histones and other chromatin proteins. We have hypothesized that the phosphorylation of protamine 1 induces protamine dimerization and that these dimers are required for efficient histone displacement. Histone displacement by protamines in late-step spermatids appears to be essential for the production of fertile sperm in man and other mammals, and the disruption of this process could provide a new approach for male contraception. As a first step toward testing this theory, we have initiated a set of in vitro experiments to determine whether or not protamine phosphorylation is essential for histone displacement. The results of these experiments, although incomplete, confirm that unphosphorylated protamine cannot effectively displace histone from DNA. Polyarginine molecules twice the size of a protamine molecule and salmine dimer were found to be more effective. These results are consistent with the theory that the disruption of protamine phosphoryation may prove to be a useful new approach for male contraception if it can be shown to facilitate or induce protamine dimerization.

\section{INTRODUCTION}

Protamines are a family of small arginine-rich proteins responsible for the packaging of DNA in most vertebrate sperm. During the final stages of sperm cell development, protamines are synthesized and deposited onto DNA, replacing the larger lysine-rich histones that package DNA in all other cells. If this process is disturbed, the sperm cells become dysfunctional. This has been observed clinically in infertile men who suffer from several fertility disorders, including oligospermia [1], fever induced infertility [2], and the round-headed sperm syndrome [3].

While it is clear that protamine plays a key role in the repackaging of DNA in late-step spermatids and sperm, we know very little about how the protein actually functions. Physical studies of the protamine-DNA complex have revealed that protamine binds to one of the grooves of DNA [4,5], neutralizing the negative charges on the phosphates in both strands and packing the DNA in a highly compact state. One protamine molecule binds to each turn of DNA. It has also been demonstrated that the serine residues in each protamine molecule are phosphorylated shortly after synthesis [6]. The reason for this phosphorylation has never been understood.

We have recently discovered that certain amino acid residues in the sequence of protamines appear to be conserved in all species, even though the amino acid sequences of different protamines as a whole are highly variable. Among the most important are the amino-terminal arginine and serine residues. The serine residues are the sites that are phosphorylated shortly after protamine synthesis, and this process results in the addition of a negatively charged phosphate group to each of several serines. The relative spacing and one to one correspondence of these residues has suggested that symmetrical dimers of protamine may form as a result of the formation of a series of salt 
bridges between the amino-terminal ends of two protamine molecules. This dimer would form shortly after synthesis and phosphorylation when the negatively charged phosphoserines are attracted to the positively charge arginines.

If this hypothesis is correct, protamine phosphorylation may represent an "Achilles' heal" of male fertility. If the phosphorylation and dimerization of protamines were to be inhibited or retarded, histone displacement would not be completed and chromatin reorganization in sperm would be disrupted. Males producing such sperm should be infertile. To test this theory, we set up an in vitro system for testing the efficiency of histone displacement by protamines and began using it to determine how effectively protamine, polyarginine and a synthetic dimer of protamine displace histone from DNA. These first experiments confirm that unphosphorylated protamines do not effectively displace histone from DNA. Polyarginine and a synthetic dimer of salmon protamine displaced histone more efficiently. Other experiments, as yet incomplete, are required to demonstrate that phosphorylated protamine will actually dimerize and effectively displace the histones in somatic chromatin and to test what effect the inhibition of this process has on male fertility.

\section{MATERIALS AND METHODS}

\section{Isolation of Liver Chromatin}

Nuclei were isolated from frozen hamster liver by homogenizing the thawed tissue in grinding media $(0.25 \mathrm{M}$ sucrose, $0.01 \mathrm{M}$ Tris $\mathrm{pH} 8,1 \mathrm{mM}$ magnesium chloride, $0.05 \mathrm{M}$ sodium bisulfite) in a Virtis homogenizer at $4^{\circ}$ $5,000 \mathrm{rpm}$ for $3 \mathrm{~min}$. The connective tissue was removed by filtration through cheesecloth, following the addition of 3 drops 2- octanol, and a crude nuclear pellet was obtained following centrifugation at $3,000 \times \mathrm{g}$ for $5 \mathrm{~min}$. The nuclei were purified by homogenizing the pellet in grinding media containing $1 \%$ Triton X-100 using a Dounce hand homogenizer to resuspend the nuclear pellet and sedimenting the nuclei by centrifugation at $3,000 \times \mathrm{g}$ for $3 \mathrm{~min}$. This process was repeated three times decreasing the centrifugation rate by $800 \times \mathrm{g}$ each time. The nuclei were washed two times in grinding medium and two times in $0.01 \mathrm{M}$ Tris pH 8,12.5 mM EDTA centrifuging at $3,000 \mathrm{Xg}$ for 3 minutes. The purified nuclei were resuspended in $0.01 \mathrm{M}$ Tris $\mathrm{pH} 8,12.5 \mathrm{mM}$ EDTA. (Total DNA concentration was determined spectrophotometrically at $260 \mathrm{~nm}$ by dissociating an aliquot of nuclei in $5 \mathrm{M}$ guanidine hydrochloride, $0.01 \mathrm{M}$ Tris pH8.)

\section{In Vitro System for Monitoring Histone Displacement}

To monitor the displacement of histones from chromatin by protamine, known amounts of bull and salmon protamine, salmine dimer or polyarginine were added to hamster liver chromatin, mixed and incubated at $4^{\circ} \mathrm{C}$ for 20 hours. The displaced histone was separated from the complex by centrifugation at $10,000 \times \mathrm{g}$ for 5 minutes. The supernatant was adjusted to $0.5 \mathrm{~N} \mathrm{HCl}$ and the purified histone TCA precipitated. The total amount of histone present in the chromatin sample was determined by fully expanding an aliquot of chromatin in 
$0.25 \mathrm{mM}$ EDTA, $0.25 \mathrm{mM}$ Tris $\mathrm{pH} 8$, and extracting the proteins with $0.5 \mathrm{~N} \mathrm{HCl}$. The relative amount of displaced histone was determined by measuring the amount of histone present in the supernatant (the histone displaced by the added protamine) and comparing it to the total amount of histone in the sample.

\section{Gel Electrophoresis and Scanning Microdensitometry}

The dried histones were dissolved in a known volume of $0.9 \mathrm{~N}$ acetic acid, $0.5 \mathrm{M}$ mercaptoethanol, $20 \%$ sucrose and the five histones were resolved by electrophoresis at $130 \mathrm{~V}$ for $3.5 \mathrm{hr}$ on acid-urea polyacrylamide gels [7]. The gels were stained in $0.1 \%$ naphthol blue black, $20 \%$ ethanol, $0.9 \mathrm{~N}$ acetic acid, destained by electrophoresis, and the amount of histone in the gels determined by scanning microdensitometry using a Shimadzu Flying Spot Microdensitometer.

\section{Synthesis and Purification of Salmine Dimer}

A dimer of salmon protamine was prepared by crosslinking the protamine (Sigma Chemical Co, St. Louis, MO) through its amino and carboxy-termini using 1-ethyl-3-(3-dimethylaminopropyl) carbodiimide (EDC). The efficiency of the crosslinking reaction was determined at different $\mathrm{pHs}$ ( $\mathrm{pH} 3$ to 7.2), different protein concentrations, and in the presence of $250 \mathrm{mM} \mathrm{NaCl}$. Protamine was dissolved in water at the appropriate concentration and the $\mathrm{pH}$ was adjusted by adding $\mu \mathrm{l}$ amounts of $0.5 \mathrm{~N} \mathrm{NaOH}$ or $\mathrm{HCl}$. A sixfold molar excess of EDC/protamine of solid EDC was added to the suspension of protamine and the reaction was carried out at $40^{\circ} \mathrm{C}$ for various lengths of time. The amount of dimer formed was assessed by polyacrylamide gel electrophoresis in $15 \%$ acidurea gels.

The protamine dimer was isolated from the resulting mixture of monomer and other multimers by HPLC on a TSK-3000 gel permeation column $(21 \mathrm{~mm} x$ $300 \mathrm{~mm}$ ) using $30 \%$ acetonitrile in water with $0.1 \%$ TFA as the mobile solvent. At a flow rate of $2 \mathrm{mls} / \mathrm{min}$, three peaks were detected at $214 \mathrm{~nm}$ wavelength with baseline separation of higher multimers from the dimer and the monomer protamine. The size and purity of the product was determined by electrophoresis in the same acid-urea gel system used to resolve the histones. The middle, dimer peak was collected and freeze-dried for further analysis.

Isolation of Naturally Phosphorylated Hamster Protamine

Late-step spermatids were isolated from hamster testes and the protamines were extracted as described previously for the mouse [8]. To minimize dephosphorylation of the protamine by endogenous phosphatases, $1 \mathrm{M}$ sodium orthovanadate was included in all the buffers as a nonspecific phosphatase inhibitor. To determine what percentage of the protamine is phosphorylated, the protein was electrophoresed in $30 \mathrm{~cm}$ acid-urea polyacrylamide gels for $30 \mathrm{hr}$ at $130 \mathrm{~V}$. Each phosphate group present in the protein adds an additional negative charge to the protein, and the electrophoretic mobility of the protein in the gel is proportionately reduced. 
Synthesis of Phosphorylated Protamine

The complete salmine molecule and its amino terminal peptide Pro-Arg4Ser 3 -Arg-Pro-Val were synthesized on the Model PS3 synthesizer (Protein Technologies) by solid phase technology using Wang resin. FMOC protection and the HOBT esters were used for coupling each amino acid in the sequence through the $\mathrm{C}$-terminal amino acid. The $\mathrm{N}$-terminal proline had a BOC group for protection. The arginines were additionally protected with the pme group but the serine hydroxyl groups were unesterified. After the terminal proline was added. the phosphitylation of the immobilized peptide was performed by a solution of 40 equivalents of dimethyl-N,N-diisopropylphosphoramidite in 50 equivalents of tetrazole. The reagents were allowed to mix for $90 \mathrm{~min}$ after which the peptide is washed and then the phospite-serines were oxidized with 20 equivalents of $\mathrm{m}$-chloroperbenzoic acid in dimethylformamide(DMF) for 30 minutes. The resin with peptide was washed $2 x$ with DMF and with methanol until dry. The peptide was decoupled from the resin with TFA, water, anisole and isopropylsilane for 90 minutes. The organics were distilled off and the peptide extracted with ether to remove residual organics. The final peptide solution was lyophilized .

\section{RESULTS AND DISCUSSION}

\section{Conservation of Phosphorylatable Sites in Protamine Molecules}

As the protein sequence alignments for vertebrate protamines show in Figure 1, all protamine molecules appear to contain a series of serine and threonine residues in the amino terminal end of the molecule. While the actual number of residues varies in the protein isolated from different species, the residues are appropriately positioned relative to neighboring arginine residues such that the negatively charged phosphate groups added to each serine could interact with an arginine residue on another molecule to form a series of salt bridges that interlock the amino-terminal ends of two protamine molecules together to form a dimer (Figure 2). This process would generate a symmetrical molecule with a DNA binding domain twice the size of the protamine monomer, one that would presumably bind to DNA with a much greater affinity. Such a positioning of neighboring protamine molecules (amino-terminus linked to amino terminus/ carboxy-terminus positioned next to carboxy-terminus) on DNA has already been demonstrated in our analyses of bull protamine disulfide bonding [9] and a dimer form of C-terminally crosslinked protamine 1 isolated from bull sperm [10].

Analysis of Histone Displacement by Unohosphorylated Protamines. Polyarginine and a Synthetic Protamine Dimer

If this theory is correct, monomeric protamines should not be able to efficiently displace histones from DNA in chromatin. In order to test this theory, an in vitro histone displacement assay was set up using isolated hamster liver chromatin. This chromatin is comprised of DNA, the lysine-rich histone $\mathrm{H1}$, and the four core histones H3, H2B, H2A and H4. In this system, protamine is added to EDTA-lysed liver nuclei (chromatin). After centrifugation, the relative amount of 
displaced histone is determined by measuring the ratio of histone present in the supernatant to the total amount of histone in the sample (as determined by acid extraction). The isolated histones are resolved by gel electrophoresis and quantified by scanning microdensitometry. This approach is straight forward and appears to yield excellent data.

The results of our in vitro histone dissociation experiments with unphosphorylated protamine, polyarginine and a synthetic salmine dimer confirm that this system can be used to monitor histone displacement from DNA. As shown in Figures 3 and 4, the unphosphorylated salmine and bull protamines dissociate the lysine rich histone $\mathrm{H} 1$ from hamster liver chromatin. The four core histones are not displaced from DNA, even at high concentrations of protamine.

To determine if a larger form of protamine, such as a dimer, can effectively displace histone, we have tested polyarginine $(\sim 10,000 \mathrm{MWt})$ and a synthetic salmon protamine dimer. The dimer of salmon protamine was prepared by chemically crosslinking the amino- and carboxy-terminal ends of protamines together with 1-Ethyl-3-(3-Dimethylaminopropyl) carbodiimide (EDC). The dimer was isolated from a mixture of monomer and dimer (Figure 5) by HPLC and preliminary experiments were conducted to test its ability to displace histones from DNA. As is shown in Figure 6, polyarginine did succeed in displacing the majority of the core histones from DNA in chromatin. The salmine dimer also appeared to displace some of the core histones, but it appeared to be less efficient than polyarginine.

\section{De Phosphorylated Protamines Dimerize and Displace Histones Efficiently?}

To further test this theory in vitro, fully phosphorylated protamine must be synthesized to provide enough material for the displacement experiments. Protamine isolated from mature sperm is usually dephosphorylated and only a relatively small percentage of the total serine residues remain in the phosphorylated state in protamines isolated from late-step spermatids. This is because nonspecific phosphatases are abundant in cell lysates, and these enzymes rapidly dephosphorylate protamines as they are being isolated. Using salmon protamine as the first model protamine for this study, we have synthesized the intact protein in such a manner that the three $\mathrm{N}$-terminal serine residues were chemically phosphorylated (Figure 7). The corresponding amino terminal peptide was also synthesized for later use in competition experiments. We are presently awaiting confirmation that the protein is phosphorylated before we test the ability of the phosphorylated protein to dimerize and to displace histones from DNA. Once the presence of the phosphates have been confirmed, the phosphorylated protamine will be tested for its ability to form dimers in solution and to displace histones from DNA. Subsequent experiments will test the ability of the phosphorylated amino-terminal peptide to inhibit the formation of dimers containing two complete protamine molecules. 
Identification of Mammalian Protamine Phosphorylation Sites

While we can test the process of protamine dimerization and histone displacement using phosphorylated salmon protamine in vitro (this protamine is easier to synthesize because it is only $60 \%$ the size of a mammalian protamine and its phosphorylation sites are known), the ultimate goal of the study is to actually test the process in vivo in mammals. However, the phosphorylation sites in mammalian protamine have not been identified. In an effort to determine the location of these phosphorylation sites, we have isolated the fully phosphorylated protamine from hamster late-step spermatids (the sequence of mouse and hamster are essentially identical, but a single hamster produces as much protamine as 150 mice - thus considerably more material can be obtained for analysis) and have begun optimizing the b-elimination reaction for converting the phosphorylated amino acids to stable derivatives so we can locate the phosphorylation sites in the protein by automated amino acid sequencing. Protamine isolations performed in the presence of a general phosphatase inhibitor, sodium orthovanadate, were found to markedly increased the yield of phosphorylated protamine extracted from late-step spermatids. Other experiments suggest that S-hydroxyethyl cysteine, the derivative formed by conducting the b-elimination reaction on proteins containing phosphoserine in the presence of mercaptoethanol, may prove suitable for analysis by automated sequencing.

\section{Future Efforts}

The primary goal of these experiments is to determine if protamine phosphorylation and dimerization are essential for male fertility. We have developed an in vitro model of chromatin condensation and histone displacement by protamine that mimicks the natural process in late-step spermatids. We are currently using this system to compare the effectiveness of histone displacement by phosphorylated and unphosphorylated protamines and a synthetic (chemically crosslinked) salmon protamine dimer.

After this model system has been well characterized and we have demonstrated that phosphorylation induced protamine dimerization facilitates histone displacement, the next step would be to investigate methods for reducing the efficiency of phospho-protamine mediated histone replacement. One approach might involve the inhibition of protamine dimerization by the addition of phosphorylated peptides which should compete in the dimerization process. Small phosphorylated peptides which are identical, or similar, to the amino-terminal end of protamine are the most likely candidates. The interactions of these inhibitors with the phosphorylated protamines could be modeled on a Silicon Graphics computer using the commercial programs QUANTA AND CHARMm (licensed from Polygen Corporation). These models could be used to design improved peptide inhibitors and these peptides could be tested in our model in vitro system.

Once an improved inhibitor has been identified, the importance of histone dimerization to fertility must be demonstrated in vivo. One approach would involve using transgenic mice. A mouse protamine 1 gene construct could be prepared containing mutations within the coding sequence that results 
in the synthesis of a truncated protamine sequence when expressed in vivo. Because this construct would be derived from a protamine gene, these peptides will be synthesized at the same time as protamine (step 11 spermatids) and only in sperm cells. If the first 13 amino acids of mouse protamine 1 were expressed, it should undergo phosphorylation and should participate in dimerization, but the absence of a DNA binding domain would prevent the complex from displacing histones from DNA.

Transgenic males containing the integrated constructs could then be bred with females to determine their fertility. Once the breeding experiments are complete and confirm the infertility of the males, their late-step spermatids and sperm would be examined to determine if the truncated peptides are present in the nucleus and if the peptides are phosphorylated in vivo. The sperm would also be examined by transmission electron and atomic force microscopy to determine how the expression of the peptides affect chromatin condensation.

\section{ACKNOWLEDGEMENTS}

This work was performed under the auspices of the U.S. Department of Energy by Lawrence Livermore National Laboratory under contract no. W-7405ENG-48 and was supported by a Lawrence Livermore National Laboratory Directed Research and Development award. 


\section{REFERENCES}

1. L. Silvestroni et al., "Histones Instead of Protamines in Terminal Germ Cells of Infertile, Oligospermic Men," Eentility and Sterility 27,12(1976).

2. D.P. Evenson, R. Balhorn, L.K. Jost, R.A. Baer and M. Corzett, "Fever Induced Unique Human Sperm Nuclear Protein is Temporally Correlated with Altered Susceptibility to DNA Denaturation in Situ and Decreased Free -SH Groups," presented at the Andrology Meeting, March 1992.

3. R. Balhorn, S. Reed and N. Tanphaichitr, "Aberrant Protamine 1/Protamine 2 Ratios in Sperm of Infertile Human Males," Experientia 44, 52 (1988).

4. M.C. Prieto-Sheaffer, A.H. Maki and R. Balhorn, "Studies of Protamine-DNA Interactions by Optically Detected Magnetic Resonance," Western Spectroscopy Conference, Pasadena, CA Oct 19-24 (1993).

5. N.V. Hud, F.P. Milanovich and R. Balhorn, "Evidence of a Novel Secondary Structure in DNA-Bound Protamine is Revealed by Raman Spectroscopy," Biochemistry 33, 7528 (1994).

6. K. Marushige, V. Ling, and G. Dixon, "Phosphorylation of Chromosomal Basic Proteins in Maturing Trout Testis," ل. Bio. Chem. 244,21(1969).

7. Histone gel electrophoresis paper

8. R. Balhorn, S. Weston, C. Thomas and A. Wyrobek, "DNA Packaging in Mouse Spermatids: Synthesis of Protamine Variants and Four Transition Proteins," Experimental Cell Res, 150, 298 (1984).

9. R. Balhorn, M. Corzett, J.A. Mazrimas and B. Watkins, "Identification of Bull Protamine Disulfides," Biochemistry 30, 175 (1991).

10. J. Mazrimas, M. Corzett and R. Balhorn, "Isolation of a Non-Disulfide Linked Protamine 1 Dimer," Presented at the Fourth International Congress of Cell Biology, Montreal, Quebec, Canada, August 14-19 (1988). 


\section{FIGURE LEGENDS}

Figure 1. Amino acid sequences for protamine molecules from various species of animals. The serine residues that are known or potential phosphorylation sites are shown in red. Single letter abbreviations are used for each amino acid.

Figure 2. Proposed dimerization scheme for phosphorylated derivatives of protamines. In this scheme, negatively charged phosphate groups on the serine (S) residues form a stable, salt bridge (shown as a vertical line) to positively charged arginine (R) residues, locking to proteins together. Question marks identify positions of possible covalent disulfide bond formation.

Figure 3. Displacement of histones from DNA in liver chromatin by salmon protamine. Three sets of samples are shown with salmon protamine added at a ratio of 0,1 and 10 arginine residues per phosphate group. The first gel in each set shows the histone displaced from the chromatin by added protamine while the second gel shows the histone remaining in the chromatin (not displaced).

Figure 4. Displacement of histones from DNA in liver chromatin by bull protamine. Shown is the histone displaced from the chromatin by added protamine at $0,0.1,0.5,1.0,2.0,8.0$ arginine residues per phosphate group.

Figure 5. HPLC' fractionation of EDC crosslinked salmon protamine monomer, dimer and trimers on a gel exclusion column.

Figure 6. Plot showing efficiency of histone displacement from DNA in liver chromatin by polyarginine $(10,000 \mathrm{MWt})$.

Figure 7. Phosphorylated salmon protamine and amino-terminal peptide synthesized for displacement and dimerization studies. The phosphorylated serine residues are shown in red. 


\begin{tabular}{|c|c|}
\hline $\begin{array}{l}\text { Bull } \\
\text { Ram } \\
\text { Boar } \\
\text { Mouse } \\
\text { Rat } \\
\text { Rabbit } \\
\text { Human } \\
\text { Opossum } \\
\text { Wallaby } \\
\text { Rooster } \\
\text { Salmon }\end{array}$ & $\begin{array}{l}\text { ARYRCCLTHSGSRCRRRRRRRCRRRRRRFGRRRRRRVCCRRYTVIRCTRQ } \\
\text { ARYRCCLTHRSRCRRRRRRCRRRRRRFGRRRRRVCCRRYTVRCTRQ } \\
\text { ARYRCCRSHSRSRCRPRRRRCRRRRRRCCPRRRRRAVCCRRYTVIRCRRC } \\
\text { ARYRCCRSKSRSRCRRRRRRCRRRRRCCRRRRRRCRRRRSYIRCKKY } \\
\text { ARYRCCRSKSRSRCRRRRRCRRRRRCCRRRRRCCRRRRSTFRCKRY } \\
\text { VRYRCCRSQSRSRCRRRRRRCRRRRRCCQRRVRKCCRRTYLRCRRY } \\
\text { ARYRCCRSQSRSRYYRQRQRSRRRRRSSCTRRRAMRCCRPRYRPRCRRH } \\
\text { ARYRRRSRSRSRSRYGRRRSSS.... } \\
\text { ARYRHSRSRSRSRYRRRRRSRYRSRRRY.... } \\
\text { ARYRSRGRSRSRTRRRRSPRSGRRSPRRRSRRRRRYGSARSRRSG.. } \\
\text { PRRRRSSSRPVRRRRRPRVSRRRRRRGGRRRR }\end{array}$ \\
\hline
\end{tabular}


Salmon

Rooster

Wallaby

Opossum

Rat/Mouse

Boar

Bull/Ram

Killer Whale

Tamarin

Human
. . RRRRRRVPRSSSRRRRP

IIIIII

PRRRRSSSRPVRRRRRR. . .

. . RRIIRRSRSRERSRYRA

$$
\begin{aligned}
& \text { I I I II I } \\
& \text { ARYRSRGRSRSRRIRR. . . }
\end{aligned}
$$

. . RRYRSRSRSRSERYRA

| | | | | |

ARYRESRSRSRSRYRR. . .

. . GIRSRSRSRSRRRYRA

| ||||||||

ARYRRRSRSRSRSRYG. . .

. . RRRCRSRSTSRCCRYRA

? \|\|\|\| ?

ARYRCCRSKSRSRCRRR. . .

...RPRCRSRSHSRCCRYRA

? I1 II ?

ARYRCCRSHSRSRCRPR. . .

. . .RRRRCRSRSHTICCRYRA

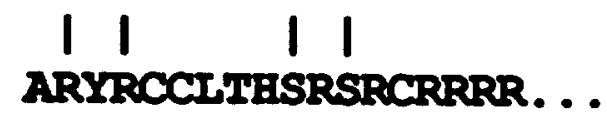

. . RPRRCRSOSPSRCRIRA

ARNRCRSPSQSRCRRPR. . .

. . RQRYCRSRSQSRCCRYRA

? 1111 ?

ARYRCCRSOSRSRCYROR. . .

. . RQRQRYYRSRSQSRCCRYRA

II II

ARYRCCRSQSRSRYYRQRQR. . . 


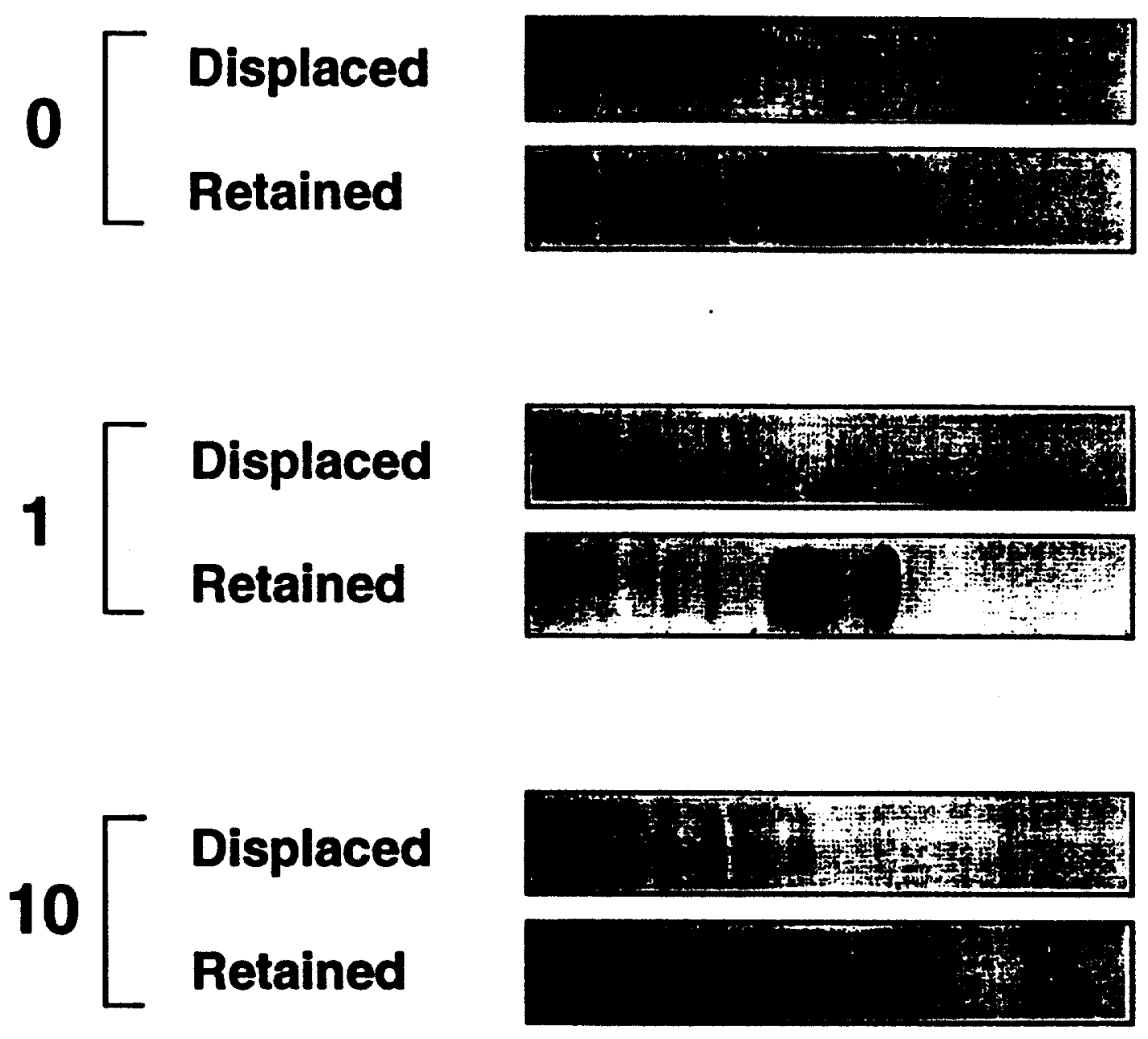

Figure 3 


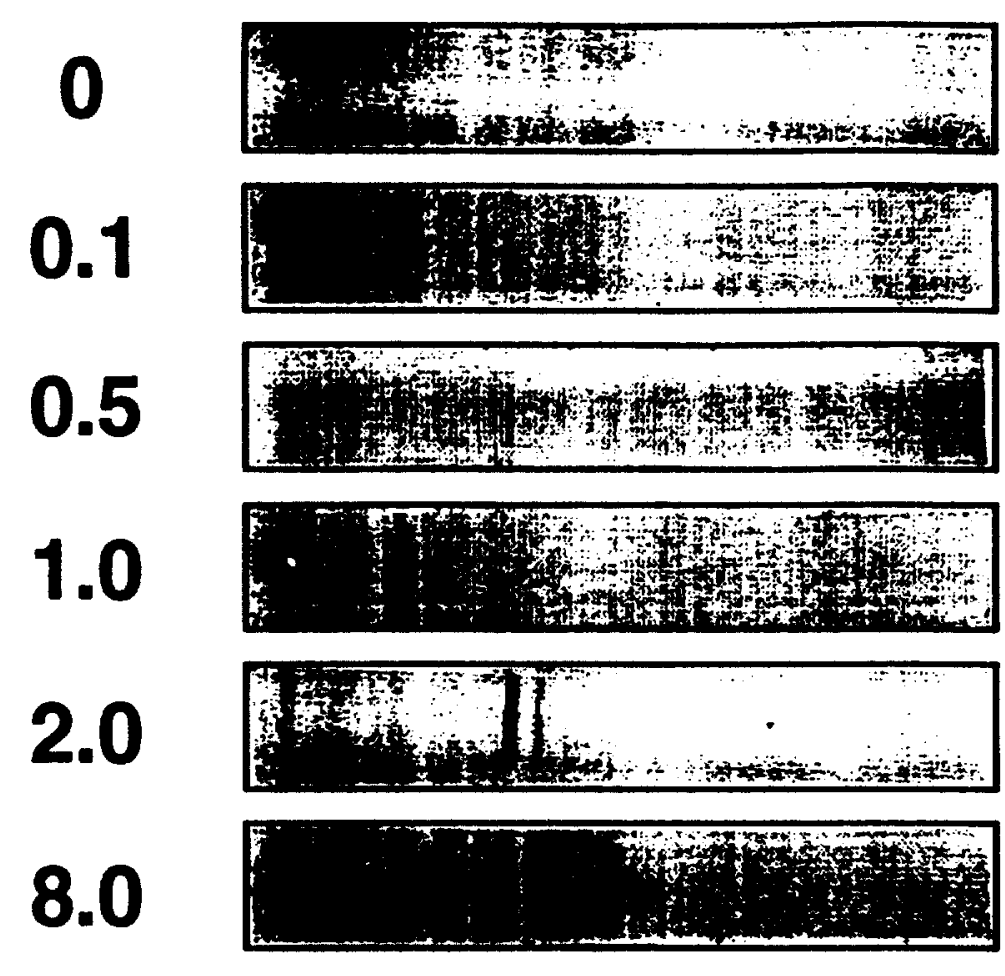

Total Histone

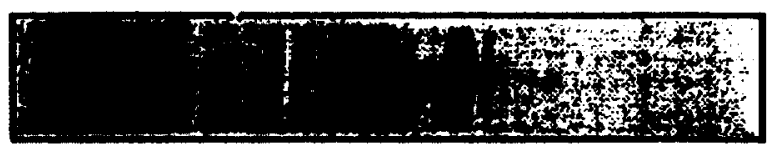




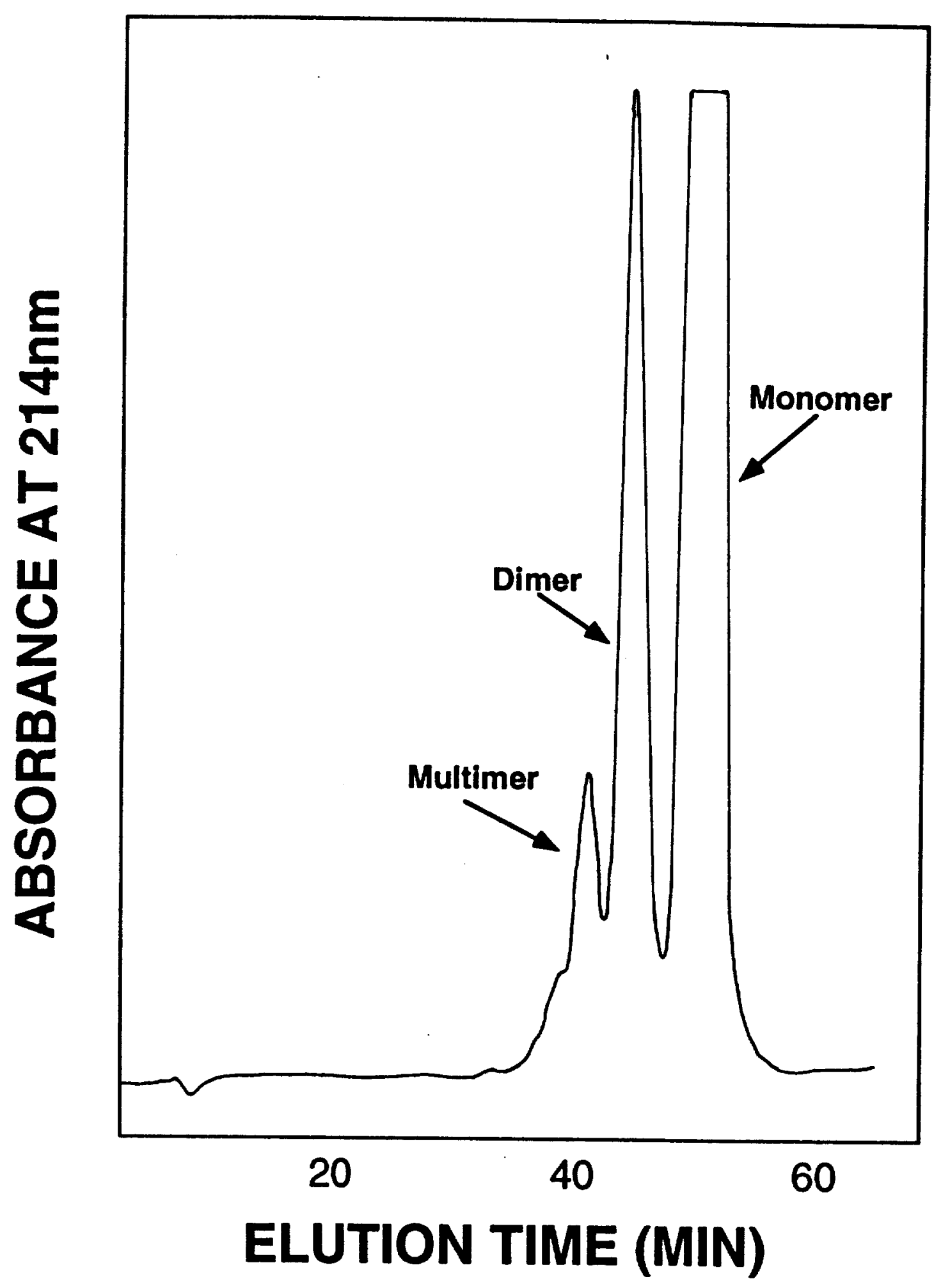

Figure 5 
POLYARGININE (10 000MW)

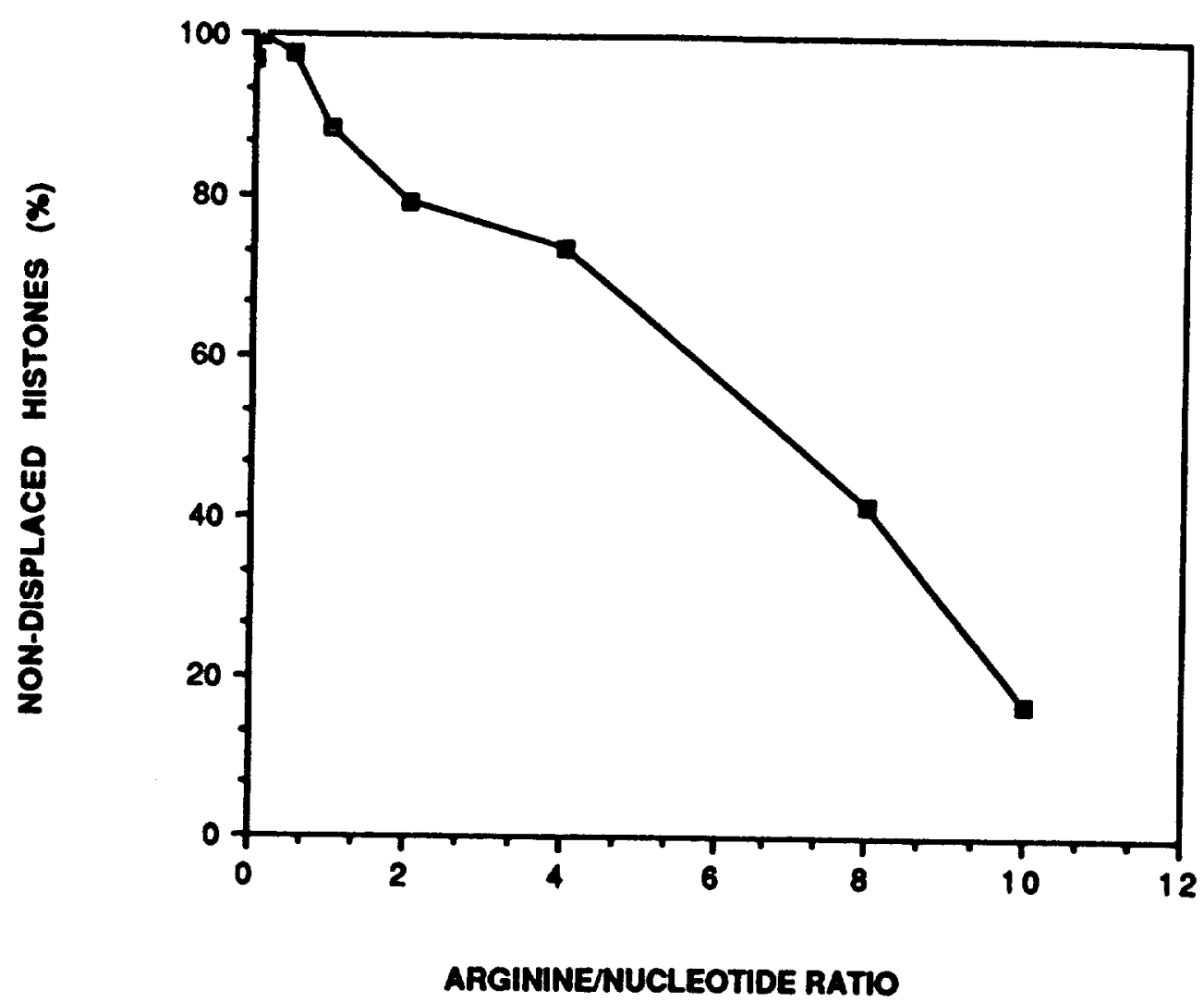

Figure 6 
Phospho-salmine:

Inhibitor peptide:
PRRRRSSSRPVRRRRRPRVSRRRRRRGGRRRR

PRRRRSSSRPV 


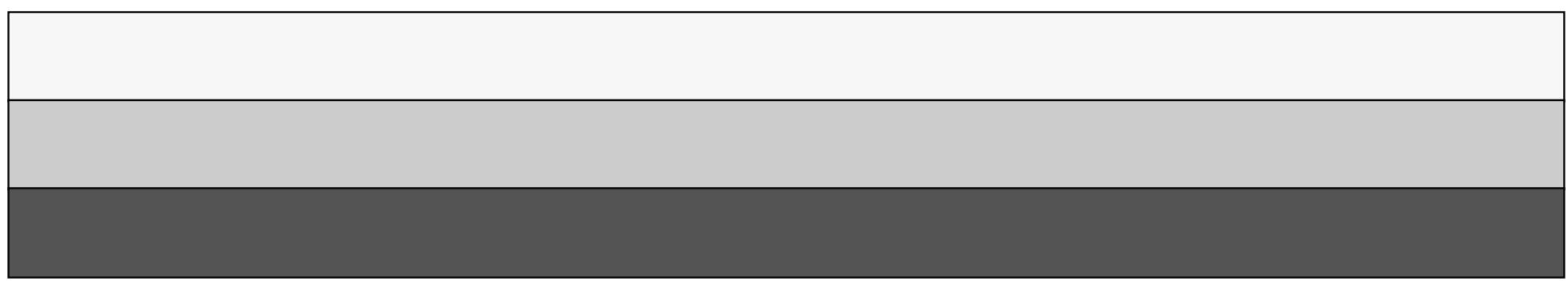

